\title{
COMMUNICATIONS
}

Comment

\section{Comment on the paper "Use of interferometric directionality for noise reduction"}

Chandra S. Vikram, FELLOW SPIE

University of Alabama in Huntsville

Center for Applied Optics

Huntsville, Alabama 35899

E-mail: vikramc@email.uah.edu

Optical Engineering 34(1), 173-182 (1995).

In a recent work, $\mathrm{Yu}$, Cha, and $\mathrm{Joo}^{1}$ describe a directional smoothing technique for reducing noise in interferograms. The idea is to replace more conventional square mask $(N \times N$ pixels) with linear $(1 \times N$ pixel) averaging parallel to the fringe direction. Thus, the blurring effects associated with the square mask can be significantly reduced, preserving the structural integrity of the fringes. This is because along the fringe counting direction (orthogonal to the fringe orientation) the resolution of one pixel is maintained. Yu, Cha, and Joo successfully applied the approach to several computergenerated as well as real interferograms.

It is worth noticing that the idea has been around for some time. For example, Robinson ${ }^{2}$ successfully applied the approach to Young's fringes in speckle photography as well as more complex fringe systems. More recently, Pomarico et $\mathrm{al}^{3}$ applied the directional averaging approach to digital speckle correlation fringes.

The contribution of $\mathrm{Yu}, \mathrm{Cha}$, and $\mathrm{Joo}^{1}$ is more general in the sense that it locally averages using $1 \times N$ pixel masks to follow complex fringe directions. In other works, ${ }^{2,3}$ the preinformation of the fringe direction is available.

In conclusion, the linear directional mask averaging approach is useful in noise reduction from interference fringes without smearing of high-density fringes. The main purpose of this comment is to give due credit to some earlier workers on the concept.

\section{References}

1. E. Yu, S. S. Cha, and W. Joo, "Use of interferometric directionality for noise reduction," Opt. Eng. 34(1), 173-182 (1995).

2. D. W. Robinson, "Automatic fringe analysis with a computer imageprocessing system,"' Appl. Opt. 22(14), 2169-2176 (1983).

3. J. Pomarico, R. Arizaga, R. Torroba, and H. Rabal, "Algorithm to compute spacing of digital speckle correlation fringes," Optik 95(3), 125127 (1994). 